\title{
METASTABLE EQUILIBRIUM OF FLUIDS WITH SURFACE TENSION*
}

\author{
BY \\ EPIFANIO G. VIRGA \\ Università di Pisa, Pisa, Italy
}

\begin{abstract}
This paper deals with the effects of the surface tension on the equilibrium of fluids. It shows that, when a fluid with an incompressible fluid inclusion is put in a fluid environment kept at constant pressure, equilibrium configurations may arise whose character of stability is affected by the sign of the perturbations of the environmental pressure. Such configurations we refer to as metastable.
\end{abstract}

1. Introduction. The classical interfacial energy between two fluids is proportional to the area of the surface of contact, the constant of proportionality being the surface tension. It is intuitive that the surface tension between two fluids contributes to the stability of their equilibrium configurations. It is the aim of this paper to exhibit fairly general conditions under which the surface tension by itself is not enough to avoid instabilities. Precisely, I shall show that the equilibrium configurations of two ideal fluids free from body forces may be metastable, no matter how strong the surface tension is. By a metastable equilibrium configuration I mean an equilibrium configuration which is not stable and which, as a structural parameter is perturbed, is changed into a stable configuration or into an unstable configuration, depending on the sign of the perturbation.

We shall study the equilibrium of an incompressible fluid inclusion $\mathscr{F}_{i}$ inside a compressible ideal fluid $\mathscr{F}$ placed in an environment $\mathscr{F}_{e}$ kept at constant pressure. We assume only that the pressure of $\mathscr{F}$ is given by a strictly decreasing function of the specific volume.

In Sec. 2 we write a variational principle for the free energy of $\mathscr{F}$ and $\mathscr{F}_{i}$ and, assuming that the regions occupied by the two fluids are sufficiently smooth, we deduce the equilibrium equations of the system as the Euler-Lagrange equations of the energy functional. As expected, in equilibrium the surfaces bounding both $\mathscr{F}$ and $\mathscr{F}_{i}$ are spheres and the interfacial pressure is consistent with the classical jump condition of Laplace.

Sec. 3 is devoted to a search for configurations of minimal energy; we interpret them as stable equilibria. To facilitate our proof of existence, we enlarge the

*Received May 29, 1986. 
admissible class of fluid regions by allowing for sets of finite perimeter. By applying the isoperimetric inequality to such sets, we find that the minima of the energy functional can be found by studying the minima of an ordinary function, which we call the lower envelope. This function provides the best lower bound to the energy functional. Moreover, all the extrema of the lower envelope are extrema of the energy functional, so that even unstable equilibrium configurations of the system are detectable by studying the lower envelope.

In Sec. 4, by exploiting the properties of the lower envelope, we prove that there is always a stable equilibrium configuration. Besides that, it is possible to find equilibrium configurations which are metastable with respect to the outside pressure. In particular, it may happen that there is an equilibrium configuration such that, as the outside pressure is increased, the configuration collapses toward a stable configuration in which $\mathscr{F}$ and $\mathscr{F}_{i}$ are inside a smaller ball, while, as the pressure is decreased, the configuration bifurcates into two equilibrium configurations, one of which is stable and the other is not.

Finally, in Sec. 5 we find that an inverse bubble may exhibit this behavior. An inverse bubble is like a soap bubble in which the soap has been replaced by a gas, and the air inside and outside the bubble by a liquid. It shows metastability if the trapped gas can be modeled as a polytropic real gas; it has only stable configurations if the gas is regarded as perfect.

2. Equilibrium equations. Consider three fluids $\mathscr{F}_{i}, \mathscr{F}$, and $\mathscr{F}_{e}$, free from body forces and arranged so that $\mathscr{F}$ surrounds $\mathscr{F}_{i}$ with $\mathscr{F}$ and $\mathscr{F}_{i}$ occupying bounded regions, and so that $\mathscr{F}_{e}$ occupies the entire space outside $\mathscr{F}$. Assume further that $\mathscr{F}_{i}$ is incompressible, that $\mathscr{F}$ is compressible but ideal, and that $\mathscr{F}$ is at the constant pressure $\pi_{e}$. Let $\tau_{i}$ be the surface tension of the interface between $\mathscr{F}_{i}$ and $\mathscr{F}$ and $\tau_{e}$ the surface tension of the interface between $\mathscr{F}$ and $\mathscr{F}$. More precisely, let $\mathscr{B}_{e}$ and $\mathscr{B}_{i} \subset \mathscr{B}_{e}$ be bounded open regions of the Euclidean three-dimensional space with $\mathbf{R}^{3}-\mathscr{B}_{e}, \mathscr{B}_{i}$, and $\mathscr{B}=\mathscr{B}_{e}-\mathscr{B}_{i}$, respectively, the regions occupied by $\mathscr{F}_{e}, \mathscr{F}_{i}$, and $\mathscr{F}$.

We assume that the mass density $\rho: \mathscr{B} \rightarrow \mathbf{R}^{+}$of $\mathscr{F}$ is of class $C^{1}$ and we write

$$
\mu=\int_{\not B} \rho
$$

for the total mass of $\mathscr{F}$. At any point $x \in \mathscr{B}, \nu(x):=1 / \rho(x)$ is the specific volume of $\mathscr{F}$, and the stored energy $\sigma(\nu)$ and the pressure $\tilde{\pi}(\nu)$ are functions of the specific volume $\nu$ with

We assume that

$$
\tilde{\pi}(\nu)=-\sigma^{\prime}(\nu)
$$

$$
\begin{aligned}
& \tilde{\pi}: \mathbf{R}^{+} \rightarrow \mathbf{R} \text { is } C^{1} \text { and strictly decreasing } \\
& \text { with } \lim _{\nu \rightarrow 0} \tilde{\pi}(\nu)=+\infty, \text { and } \lim _{\nu \rightarrow \infty} \tilde{\pi}(\nu)=0 .
\end{aligned}
$$

These assumptions ensure that $\sigma$ is strictly convex on $(0,+\infty)$.

We also use the following notation: for any Borel set $\mathscr{S}$ of $\mathbf{R}^{3}, \operatorname{vol}(\mathscr{S})$ is the Lebesgue measure of $\mathscr{P}$, while area $(\partial \mathscr{S})$ is the two-dimensional Hausdorff measure 
of its boundary. Further, we use the term regular domain for any open, bounded, and simply connected set $\mathscr{S}$ of $\mathbf{R}^{3}$ with $\partial \mathscr{S}$ a surface of class $C^{2}$.

Let $\mathscr{B}_{e}$ and $\mathscr{B}_{i} \subset \mathscr{B}_{e}$ be regular domains. Then the energy functional for $\mathscr{F}$ and $\mathscr{F}_{i}$ is defined by

$$
\mathscr{E}\left[\mathscr{B}_{e}, \mathscr{B}_{i} ; \rho\right]:=\int_{\mathscr{B}} \rho \sigma(1 / \rho)+\tau_{e} \operatorname{area}\left(\partial \mathscr{B}_{e}\right)+\pi_{e} \operatorname{vol}\left(\mathscr{B}_{e}\right)+\tau_{i} \operatorname{area}\left(\partial \mathscr{B}_{i}\right),
$$

with $\rho$ consistent with the constraint $(1)$, and $\mathscr{B}_{i}$ such that

$$
\operatorname{vol}\left(\mathscr{B}_{i}\right)=\beta_{i}
$$

with $\beta_{i}$ a fixed positive constant.

We now deduce the Euler-Lagrange equations of the functional (3). To begin with, we remove the constraint (4) by introducing the modified energy functional

$$
\mathscr{E}^{*}\left[\mathscr{B}_{e}, \mathscr{B}_{i} ; \rho\right]:=\mathscr{E}\left[\mathscr{B}_{e}, \mathscr{B}_{i} ; \rho\right]+\pi_{i}\left(\beta_{i}-\operatorname{vol}\left(\mathscr{B}_{i}\right)\right),
$$

where $\pi_{i}$ is a Lagrange multiplier representing the unknown pressure inside $\mathscr{F}_{i}$.

Let $f: \mathscr{B}_{e} \rightarrow \mathbf{R}^{3}$ be a regular deformation of $\mathscr{B}_{e}$, i.e., a one-to-one function of class $C^{2}$ preserving the local orientation of $\mathbf{R}^{3}$. Then, both $f\left(\mathscr{B}_{i}\right)$ and $f\left(\mathscr{B}_{e}\right)$ are regular domains, $f\left(\mathscr{B}_{i}\right) \subset f\left(\mathscr{B}_{e}\right)$ and $f(\mathscr{B})=f\left(\mathscr{B}_{e}\right)-f\left(\mathscr{B}_{i}\right)$. We denote by $\mathbf{F}(x)$ the gradient of $f$ at $x \in \mathscr{B}_{e}$, by $\mathbf{F}^{-\mathrm{T}}(x)$ the inverse of the transpose of $\mathbf{F}(x)$, and by

$$
\mathbf{F}^{*}(x)=(\operatorname{det} \mathbf{F}) \mathbf{F}^{-\mathrm{T}}
$$

the adjoint of $\mathbf{F}(x)$. If $\mathbf{n}_{e}(x)$ is the unit outward normal of $\mathscr{B}_{e}$ at point $x \in \partial \mathscr{B}_{e}$ and $\mathbf{m}_{e}(y)$ is the unit outward normal of $f\left(\mathscr{B}_{e}\right)$ at point $y \in f\left(\partial \mathscr{B}_{e}\right)$, then (see, e.g., [Gu],p. 54) as

$$
\mathbf{m}_{e}(f(x))=\left|\mathbf{F}^{*}(x) \mathbf{n}_{e}\right|^{-1} \mathbf{F}^{*}(x) \mathbf{n}_{e}, \quad \text { for every } x \in \partial \mathscr{B}_{e},
$$

we have

$$
\begin{aligned}
& \operatorname{area}\left(\partial f\left(\mathscr{B}_{e}\right)\right)=\int_{\partial f\left(\mathscr{B}_{c}\right)} \mathbf{m}_{e} \cdot \mathbf{m}_{e}=\int_{\partial \mathscr{B}_{c}}\left(\mathbf{F}^{*} \mathbf{n}_{e} \cdot \mathbf{F}^{*} \mathbf{n}_{e}\right)^{1 / 2}, \\
& \operatorname{vol}\left(f\left(\mathscr{B}_{e}\right)\right)=(1 / 3) \int_{\partial f\left(\mathscr{B}_{c}\right)} \mathbf{m}_{e} \cdot y=(1 / 3) \int_{\partial \mathscr{B}_{c}} \mathbf{F}^{*} \mathbf{n}_{e} \cdot f .
\end{aligned}
$$

Similarly,

$$
\begin{aligned}
\operatorname{area}\left(\partial f\left(\mathscr{B}_{i}\right)\right) & =\int_{\partial \mathscr{B}_{i}}\left(\mathbf{F}^{*} \mathbf{n}_{i} \cdot \mathbf{F}^{*} \mathbf{n}_{i}\right)^{1 / 2}, \\
\operatorname{vol}\left(f\left(\mathscr{B}_{i}\right)\right) & =(1 / 3) \int_{\partial \mathscr{B}_{i}} \mathbf{F}^{*} \mathbf{n}_{i} \cdot f
\end{aligned}
$$

where $\mathbf{n}_{i}$ is the unit outward normal field on $\partial \mathscr{B}_{i}$. Let

$$
\rho_{f}(f(x))=\rho(x) / \operatorname{det} \mathbf{F}(x), \quad \text { for every } x \in \mathscr{B} .
$$

Then, by (8),

$$
\int_{f(\mathscr{B})} \rho_{f}=\int_{\mathscr{B}} \rho,
$$


and $\rho_{f}$ fulfills constraint $(1)$ whenever $\rho$ does. Thus, if $\left(\mathscr{B}_{\ell}, \mathscr{B}_{i} ; \rho\right)$ is an admissible triplet for the functional $\mathscr{E}^{*}$, then $\left(f\left(\mathscr{B}_{e}\right), f\left(\mathscr{B}_{i}\right) ; \rho_{f}\right)$ is also. This enables us to define a class of variations of $\mathscr{E}^{*}$. Let $\varepsilon \in\left[0, \varepsilon_{0}\right]$ with $\varepsilon_{0}$ sufficiently small, let $v: \mathscr{B}_{e} \rightarrow \mathbf{R}^{3}$ be any function of class $C^{2}$, and let

$$
f(x)=x+\varepsilon v(x), \quad \text { for every } x \in \mathscr{B}_{e},
$$

so that $f$, as $\mathbf{F}$, and $\mathbf{F}^{*}$ depend on the parameter $\varepsilon$. Now evaluate $\mathscr{E}^{*}$ at $\left(f\left(\mathscr{B}_{e}\right), f\left(\mathscr{B}_{i}\right) ; \rho_{f}\right)$ with $f$ as in $(10)$ :

$$
\begin{aligned}
\mathscr{E}^{*}\left[f\left(\mathscr{B}_{e}\right), f\left(\mathscr{B}_{i}\right) ; \rho_{f}\right]= & \int_{\mathscr{B}} \rho \sigma(\operatorname{det} \mathbf{F} / \rho)+\tau_{e} \int_{\partial \mathscr{B}_{e}}\left(\mathbf{F}^{*} \mathbf{n}_{e} \cdot \mathbf{F}^{*} \mathbf{n}_{e}\right)^{1 / 2} \\
& +\left(\pi_{e} / 3\right) \int_{\partial \mathscr{B}_{e}} \mathbf{F}^{*} \mathbf{n}_{e} \cdot f+\tau_{i} \int_{\partial \mathscr{B}_{i}}\left(\mathbf{F}^{*} \mathbf{n}_{i} \cdot \mathbf{F}^{*} \mathbf{n}_{i}\right)^{1 / 2} \\
& +\pi_{i}\left(\beta_{i}-(1 / 3) \int_{\partial \mathscr{B}_{i}} \mathbf{F}^{*} \mathbf{n}_{i} \cdot f\right) .
\end{aligned}
$$

The right-hand side of (11) is a function of $\varepsilon$ whose first derivative at $\varepsilon=0$ is the first variation of $\mathscr{E}^{*}$ at $\left(\mathscr{B}_{e}, \mathscr{B}_{i} ; \rho\right)$ taken along a path depending on $\mathbf{v}$ :

$$
\delta \mathscr{E}^{*}\left(\mathscr{B}_{e}, \mathscr{B}_{i} ; \rho\right)[v]:=\left.D_{\varepsilon} \mathscr{E}^{*}\left[f\left(\mathscr{B}_{e}\right), f\left(\mathscr{B}_{i}\right) ; \rho_{f}\right]\right|_{\varepsilon=0} .
$$

By (10) and (11),

$$
\begin{aligned}
\delta \mathscr{E}^{*}\left(\mathscr{B}_{e}, \mathscr{B}_{i} ; \rho\right)[\mathbf{v}]= & \int_{\mathscr{B}} \sigma^{\prime}(1 / \rho) \operatorname{div} \mathbf{v}+\tau_{e} \int_{\partial \mathscr{B}_{e}} \mathbf{n}_{e} \cdot \mathbf{V} \mathbf{n}_{e}+\left(\pi_{e} / 3\right) \int_{\partial \mathscr{B}_{e}} \mathbf{v} \cdot \mathbf{n}_{e} \\
& +\left(\pi_{e} / 3\right) \int_{\partial \mathscr{B}_{e}} \mathbf{n}_{e} \cdot \mathbf{V} x+\tau_{i} \int_{\partial \mathscr{B}_{i}} \mathbf{n}_{i} \cdot \mathbf{V} \mathbf{n}_{i} \\
& -\left(\pi_{i} / 3\right) \int_{\partial \mathscr{B}_{i}} \mathbf{v} \cdot \mathbf{n}_{i}-\left(\pi_{i} / 3\right) \int_{\partial \mathscr{B}_{i}} \mathbf{n}_{i} \cdot \mathbf{V} x,
\end{aligned}
$$

where $\mathbf{V}:=(\operatorname{div} \mathbf{v}) \mathbf{I}-\nabla \mathbf{v}, \mathbf{I}$ denotes the identity tensor, and $\nabla \mathbf{v}$ is the gradient of $\mathbf{v}$. By integrating the first integral on the right-hand side of (13) by parts and applying Gauss's theorem, we obtain

$$
\begin{aligned}
\delta_{\mathscr{E}^{*}}\left(\mathscr{B}_{e}, \mathscr{B}_{i} ; \rho\right)[\mathbf{v}]= & \int_{\mathscr{B}} \rho^{-2} \sigma^{\prime \prime}(1 / \rho) \nabla \rho \cdot \mathbf{v} \\
& +\tau_{e} \int_{\partial \mathscr{B}_{e}} \mathbf{n}_{e} \cdot \mathbf{V} \mathbf{n}_{e}+\int_{\partial \mathscr{B}_{e}}\left(\pi_{e}-\tilde{\pi}\right) \mathbf{v} \cdot \mathbf{n}_{e} \\
& +\tau_{i} \int_{\partial \mathscr{B}_{i}} \mathbf{n}_{i} \cdot \mathbf{V n}_{i}+\int_{\partial \mathscr{B}_{i}}\left(\tilde{\pi}-\pi_{i}\right) \mathbf{v} \cdot \mathbf{n}_{i} .
\end{aligned}
$$

The following scalar field on $\partial \mathscr{B}_{e}$ :

$$
\mathbf{n}_{e} \cdot \mathbf{V} \mathbf{n}_{e}=\mathbf{n}_{e} \cdot[(\operatorname{div} \mathbf{v}) \mathbf{I}-\nabla \mathbf{v}] \mathbf{n}_{e}=\left(\mathbf{I}-\mathbf{n}_{e} \otimes \mathbf{n}_{e}\right) \cdot \nabla \mathbf{v}=: \operatorname{div}_{e} \mathbf{v},
$$

which involves only the tangential derivatives of $\mathbf{v}$, defines the surface divergence of v. As $\partial \mathscr{B}_{e}$ is of class $C^{2}$, we have (see, e.g., [GM], Eq. (2.9))

$$
\operatorname{div}_{e} \mathbf{v}=\operatorname{div}_{e} \mathbf{v}_{e}+2 \kappa_{e} \mathbf{v} \cdot \mathbf{n}_{e},
$$


where $\mathbf{v}_{e}:=\left(\mathbf{I}-\mathbf{n}_{e} \otimes \mathbf{n}_{e}\right) \mathbf{v}$ denotes the tangential component of $\mathbf{v}$ on $\partial \mathscr{B}_{e}$, and $\kappa_{e}$ is the mean curvature of $\partial \mathscr{B}_{e}$. We accept the convention that $\kappa_{e}$ is negative if the centers of curvature of $\partial \mathscr{B}_{e}$ lie outside $\mathscr{B}_{e}$. Similarly, at any point of $\partial \mathscr{B}_{i}$ we define

$$
\mathbf{n}_{i} \cdot \mathbf{V n}_{i}=: \operatorname{div}_{i} \mathbf{v}
$$

so that

$$
\operatorname{div}_{i} \mathbf{v}=\operatorname{div}_{i} \mathbf{v}_{i}+2 \kappa_{i} \mathbf{v} \cdot \mathbf{n}_{i},
$$

where $\kappa_{i}$ is the mean curvature of $\partial \mathscr{B}_{i}$ and $\mathbf{v}_{i}:=\left(\mathbf{I}-\mathbf{n}_{i} \otimes \mathbf{n}_{i}\right) \mathbf{v}$. By inserting (16) and (18) in (14) and applying Stokes' theorem, we easily deduce that $\delta \mathscr{E}^{*}\left(\mathscr{B}_{e}, \mathscr{B}_{i} ; \rho\right)[\mathbf{v}]=$ 0 , for every $\mathbf{v}$, if and only if

$$
\nabla \rho=0, \quad \tilde{\pi}(1 / \rho)-\pi_{e}=2 \kappa_{e} \tau_{e}, \quad \pi_{i}-\tilde{\pi}(1 / \rho)=2 \kappa_{i} \tau_{i}
$$

Note that Equations $(19)_{2}$ and $(19)_{3}$ are simply the classical jump condition of Laplace (see, e.g., [La], p. 456) for the pressure at the interface between two fluids.

Equation $(19)_{1}$ implies that $\rho$ must be constant, and thus, by virtue of $(19)_{2,3}$, renders the mean curvature of both $\partial \mathscr{B}_{i}$ and $\partial \mathscr{B}_{e}$ constant.

Alexandrov proved in [A1] that if a $C^{2}$ hypersurface bounding a domain of $\mathbf{R}^{n}$ has constant mean curvature, then it is a hypersphere. ${ }^{1}$ By applying Alexandrov's theorem to $\partial \mathscr{B}_{i}$ and $\partial \mathscr{B}_{e}$, we conclude that $\mathscr{E}^{*}$ takes extreme values on balls $\tilde{\mathscr{B}}_{e}$ and $\tilde{\mathscr{B}}_{i}$, whose radii we denote by $\alpha_{e}$ and $\alpha_{i}$, respectively. As $\tilde{\mathscr{B}}_{e} \supset \tilde{\mathscr{B}}_{i}$, then $\alpha_{e}>\alpha_{i}$. $\tilde{\mathscr{B}}_{i}$ is an admissible set for $\mathscr{E}$ only if it satisfies constraint (4), that is, only if

$$
\alpha_{i}=\left(\frac{3 \beta_{i}}{4 \pi}\right)^{1 / 3} \text {. }
$$

Equations $(19)_{1,2}$ and (1) imply that $\mathscr{E}$ attains extrema on triplets $\left(\tilde{\mathscr{B}}_{e}, \tilde{\mathscr{B}}_{i} ; \tilde{\rho}\right)$ in which $\tilde{\rho}$ is the function

$$
\tilde{\rho}(x)=\rho_{0}, \quad \text { for every } x \in \tilde{\mathscr{B}}:=\tilde{\mathscr{B}}_{e}-\tilde{\mathscr{B}}_{i}
$$

and

$$
\begin{gathered}
\rho_{0}=\frac{3 \mu}{4 \pi}\left(\alpha_{e}^{3}-\alpha_{i}^{3}\right)^{-1}, \\
\tilde{\pi}\left(1 / \rho_{0}\right)-\pi_{e}=2 \tau_{e} / \alpha_{e} .
\end{gathered}
$$

Any couple $\left(\rho_{0}, \alpha_{e}\right)$ solving (22) and (23) defines a double infinity of equilibrium configurations for $\mathscr{F}$ and $\mathscr{F}_{i}$, as the ball $\tilde{\mathscr{B}}_{e}$ can be located everywhere in the space and the ball $\tilde{\mathscr{B}}_{i}$ everywhere inside $\tilde{\mathscr{B}}_{e}$. Finally, for any equilibrium configuration, $(19)_{3}$ gives the pressure of $\mathscr{F}_{i}, \pi_{i}$. It is easily seen that $\pi_{i}$ is greater than both the pressure inside $\mathscr{F}_{i}$ and the outside pressure $\pi_{e}$.

If the function $\mathbf{v}$ defined in (10) has compact support in $\tilde{\mathscr{B}}$, it is a simple matter to compute the second variation of $\mathscr{E}^{*}$ at $\left(\tilde{\mathscr{B}}_{\rho}, \tilde{\mathscr{B}}_{i} ; \tilde{\rho}\right)$. Indeed, then only the first

${ }^{1}$ This theorem was formerly proved by Liebman [Li] for a compact, strictly convex surface of $\mathbf{R}^{3}$. Other proofs of this theorem, under more general assumptions, can be found in [H], [A2], [R1], and [R2]. For a brief historical outline of this subject we refer to [O], Sec. 2 . 
integral on the right-hand side of (13) affects the variation of $\delta \mathscr{E}^{*}$, and

$$
\delta^{2} \mathscr{E}^{*}\left(\tilde{\mathscr{B}}_{e}, \tilde{\mathscr{B}}_{i} ; \tilde{\rho}\right)[\mathbf{v}]=\rho_{0}^{-1} \sigma^{\prime \prime}\left(1 / \rho_{0}\right) \int_{\mathscr{B}}(\operatorname{div} \mathbf{v})^{2}, \quad \text { for any } \mathbf{v} \in C_{0}^{2}(\tilde{\mathscr{B}}) .
$$

Since $\sigma$ is a strictly convex function, the right-hand side of (24) is nonnegative for any nonzero $\mathbf{v}$. This shows that any equilibrium configuration is materially stable, as the energy does not decrease for every local perturbation of the density $\tilde{\rho}$.

3. Energy minimizers. In this section, by applying to sets of $\mathbf{R}^{3}$ a classical result obtained by De Giorgi [DG3] within the theory of minimal surfaces [DG1,2], I shall prove that the energy functional $\mathscr{E}$ has minimizers when $\mathscr{B}_{e}$ and $\mathscr{B}_{i}$ belong to the class of sets with finite perimeter.

Let $\mathscr{C}$ be a Borel set of $\mathbf{R}^{3}$. Then the perimeter of $\mathscr{C}$ (see, e.g., [Gi], p. 5) is the scalar

$$
\operatorname{pm}(\mathscr{C}):=\sup \left\{\int_{\mathscr{C}} \operatorname{div} g\right\}
$$

with the supremum taken on all functions $g: \mathbf{R}^{3} \rightarrow \mathbf{R}^{3}$ of class $C^{1}$ with compact support in $\mathbf{R}^{3}$, such that $|g(x)| \leq 1$, for every $x \in \mathbf{R}^{3}$. $^{2}$

If $\operatorname{pm}(\mathscr{C})<+\infty$, then $\mathscr{C}$ is also called a Caccioppoli set. It is not difficult to prove that the perimeter of any Caccioppoli set $\mathscr{C}$ of $\mathbf{R}^{3}$ with $C^{2}$ boundary is the two-dimensional Hausdorff measure of $\partial \mathscr{C}$; in particular, if $\mathscr{C}$ is a regular domain of $\mathbf{R}^{3}$, then

$$
\operatorname{pm}(\mathscr{C})=\operatorname{area}(\partial \mathscr{C})
$$

The family of all Borel sets of $\mathbf{R}^{3}$, endowed with the distance

$$
\operatorname{dist}\left(\mathscr{C}, \mathscr{C}^{\prime}\right):=\operatorname{vol}\left(\mathscr{C} \cup \mathscr{C}^{\prime}-\mathscr{C} \cap \mathscr{C}^{\prime}\right),
$$

is a metric space. We say that two sets $\mathscr{C}$ and $\mathscr{C}^{\prime}$ are equivalent when $\operatorname{dist}\left(\mathscr{C}, \mathscr{C}^{\prime}\right)=0$; two equivalent sets have the same perimeter. It is easy to verify that if both $\mathscr{C}$ and $\mathscr{C}^{\prime}$ have finite volume, then

$$
\operatorname{dist}\left(\mathscr{C}, \mathscr{C}^{\prime}\right) \geq\left|\operatorname{vol}(\mathscr{C})-\operatorname{vol}\left(\mathscr{C}^{\prime}\right)\right|
$$

so that if $\operatorname{dist}\left(\mathscr{C}, \mathscr{C}^{\prime}\right)$ approaches zero, then $\operatorname{vol}(\mathscr{C})$ approaches $\operatorname{vol}\left(\mathscr{C}^{\prime}\right)$.

In the class of finite-perimeter sets the isoperimetric inequality holds:

$$
\operatorname{pm}(\mathscr{C}) \geq(4 \pi)^{1 / 3}(3 \operatorname{vol}(\mathscr{C}))^{2 / 3},
$$

with equality only when $\mathscr{C}$ is (or is equivalent to) a ball. ${ }^{3}$

Let $\mathscr{B}_{e}$ and $\mathscr{B}_{i} \subset \mathscr{B}_{e}$ be sets of finite perimeter; we write the energy functional of $\mathscr{F}_{i}$ and $\mathscr{F}$ as

$$
\mathscr{E}\left[\mathscr{B}_{e}, \mathscr{B}_{i} ; \rho\right]=\int_{\mathscr{B}} \rho \sigma(1 / \rho)+\tau_{e} \operatorname{pm}\left(\mathscr{B}_{e}\right)+\pi_{e} \operatorname{vol}\left(\mathscr{B}_{e}\right)+\tau_{i} \operatorname{pm}\left(\mathscr{B}_{i}\right),
$$

\footnotetext{
${ }^{2}$ De Giorgi used in [DG1] a different definition of perimeter. It is possible to show (see [Gi], p. 13) that the original De Giorgi definition is equivalent to (1).

${ }^{3}$ See [DG3], Sec. 4, Thm. I. A different proof of (5), resorting to Sobolev inequality, can be found in [FF], Sec. 6 (see also [FR] and [T]).
} 
with the constraints

$$
\begin{gathered}
\int_{\mathscr{B}} \rho=\mu, \\
\operatorname{vol}\left(\mathscr{B}_{i}\right)=\beta_{i}
\end{gathered}
$$

(cf. (2.3), (2.1), and (2.4)). Suppose that $\rho$ is any function which renders the energy finite. As $\sigma$ is strictly convex, the mapping $\rho \mapsto \rho \sigma(1 / \rho)$ is also strictly convex. By applying Jensen's inequality (cf., e.g., [HLP], p. 150) to the first integral on the right-hand side of (6), and inequality (5) to the last, and then using (7) and (8), we get

$$
\begin{aligned}
\mathscr{E}\left[\mathscr{B}_{e}, \mathscr{B}_{i} ; \rho\right] \geq \mathscr{E}_{*}\left[\mathscr{B}_{e}\right]:= & \mu \sigma\left(\left(\operatorname{vol}\left(\mathscr{B}_{e}\right)-\beta_{i}\right) / \mu\right)+\tau_{e} \operatorname{pm}\left(\mathscr{B}_{e}\right) \\
& +\pi_{e} \operatorname{vol}\left(\mathscr{B}_{e}\right)+\tau_{i}(4 \pi)^{1 / 3}\left(3 \beta_{i}\right)^{2 / 3} .
\end{aligned}
$$

In $(9)_{1}$ equality prevails only if $\rho$ is the constant $\mu /\left(\operatorname{vol}\left(\mathscr{B}_{e}\right)-\beta_{i}\right)$ and $\mathscr{B}_{i}$ is a ball whose radius $\alpha_{i}$ is given by (2.20).

This shows that, for every $\mathscr{B}_{e}$ such that $\operatorname{vol}\left(\mathscr{B}_{e}\right) \geq \beta_{i}, \mathscr{E}_{*}\left[\mathscr{B}_{e}\right]$ is the absolute minimum of $\mathscr{E}\left[\mathscr{B}_{e}, \mathscr{B}_{i} ; \rho\right]$ with respect to $\mathscr{B}_{i}$ and $\rho$.

By applying inequality (5) to $\mathrm{pm}\left(\mathscr{B}_{e}\right)$, it follows from $(9)_{2}$ that

$$
\begin{aligned}
\mathscr{E}_{*}\left[\mathscr{B}_{e}\right] \geq & \mu \sigma\left(4 \pi\left(\alpha^{3}-\alpha_{i}^{3}\right) /(3 \mu)\right)+\tau_{e} 4 \pi \alpha^{2} \\
& +4 \pi \pi_{e} \alpha^{3} / 3+\tau_{i} 4 \pi \alpha_{i}^{2}=: \varphi\left(\alpha ; \pi_{e}\right),
\end{aligned}
$$

with equality only if $\mathscr{B}_{e}$ is a ball of radius $\alpha>\alpha_{i}$.

For every $\pi_{e}>0$, we call the mapping $\alpha \mapsto \varphi\left(\alpha ; \pi_{e}\right)$ the lower envelope of $\mathscr{E}_{*}$. We now show that

(i) every extremum of the lower envelope of $\mathscr{E}_{*}$ is an extremum of $\mathscr{E}$ in the class of regular domains;

(ii) every local minimum of $\mathscr{E}_{*}$ with respect to the topology induced by (3) is a local minimum of the function $\varphi\left(\cdot ; \pi_{e}\right)$, and vice versa.

Indeed, by (2.2), the roots $\alpha_{e}>\alpha_{i}$ of

$$
\varphi_{\alpha}^{\prime}\left(\alpha ; \pi_{e}\right)=4 \pi \alpha\left[\alpha \sigma^{\prime}\left(4 \pi\left(\alpha^{3}-\alpha_{i}^{3}\right) /(3 \mu)\right)+2 \tau_{e}+\pi_{e} \alpha\right]=0,
$$

are the same as the roots of (2.22) and (2.23); this proves (i). To prove (ii), recall first that, by $(10)$, every nonspherical domain gives the functional $\mathscr{E}_{*}$ a value greater than the one attained on a ball with the same volume. Let $\tilde{\mathscr{B}}_{e}$ be a ball of radius $\alpha_{e}$ which minimizes $\mathscr{E}_{*}$. By (4), on any sequences of balls approximating $\tilde{\mathscr{B}}_{e}$ with respect to (3), the radius tends to $\alpha_{e}$, and the energy $\varphi\left(\cdot ; \pi_{e}\right)$ decreases to $\varphi\left(\alpha_{e} ; \pi_{e}\right)$. Then $\varphi\left(\cdot ; \pi_{e}\right)$ has a minimum at $\alpha=\alpha_{e}$. On the other hand, if $\alpha_{e}$ is a local minimizer of $\varphi\left(\cdot ; \pi_{e}\right)$, then $\tilde{\mathscr{B}}_{e}$ is a local minimizer of $\mathscr{E}_{*}$, since, by (4) and (10), any set approximating $\tilde{\mathscr{B}}_{e}$ has volume close to the volume of $\tilde{\mathscr{B}}_{e}$ and energy greater than $\mathscr{E}_{*}\left[\tilde{\mathscr{B}}_{e}\right]$.

We say that the ball $\tilde{\mathscr{B}}_{e}$ is

(i) stationary if $\mathscr{E}_{*}\left[\tilde{\mathscr{B}}_{e}\right]$ is an extremum of $\mathscr{E}$ in the class of regular domains;

(ii) stable if $\mathscr{E}_{*}\left[\tilde{\mathscr{B}}_{e}\right]$ is a local minimum of $\mathscr{E}_{*}$ with respect to (3);

(iii) unstable if it is stationary, but $\mathscr{E}_{*}\left[\tilde{\mathscr{B}}_{e}\right]$ is not a local minimum;

(iv) metastable with respect to a parameter if it is stationary for a given value of this parameter and, when the parameter is perturbed, the character of stability is sensitive to the sign of the perturbation. 
4. Metastable equilibrium configurations. We now apply the results of Sec. 3 to show that a ball $\tilde{\mathscr{B}}_{e}$ may be metastable with respect to the outside pressure $\pi_{e}$.

Consider the function (cf. (3.11))

$$
\varphi_{\alpha}^{\prime}\left(\alpha ; \pi_{e}\right)=4 \pi \alpha\left[\alpha \sigma^{\prime}\left(4 \pi\left(\alpha^{3}-\alpha_{i}^{3}\right) /(3 \mu)\right)+2 \tau_{e}+\pi_{e} \alpha\right], \quad \text { for } \alpha>\alpha_{i} .
$$

It follows from $(\mathrm{H})$ that

$$
\lim _{\alpha \rightarrow \alpha_{i}} \varphi_{\alpha}^{\prime}\left(\alpha ; \pi_{e}\right)=-\infty, \quad \lim _{\alpha \rightarrow+\infty} \varphi_{\alpha}^{\prime}\left(\alpha ; \pi_{e}\right)=+\infty .
$$

Thus, for every $\pi_{e}$, the equation

$$
\varphi_{\alpha}^{\prime}\left(\alpha ; \pi_{e}\right)=0
$$

has at least one root $\alpha_{e}>\alpha_{i}$ such that $\varphi_{\alpha}^{\prime \prime}\left(\alpha_{e} ; \pi_{e}\right)>0$. Thus there is always a stable ball $\tilde{\mathscr{B}}_{e}$.

Suppose that for a given value of $\pi_{e}$ the equations (3) and

$$
\varphi_{\alpha}^{\prime \prime}\left(\alpha ; \pi_{e}\right)=0
$$

have a root at $\alpha=\alpha^{*}$, and let

$$
\varphi_{\alpha}^{\prime \prime \prime}\left(\alpha^{*} ; \pi_{e}\right) \neq 0 .
$$

Then, as a consequence of (2), there is at least one other root of (3), say $\alpha_{*}$, such that

$$
\varphi_{\alpha}^{\prime \prime \prime}\left(\alpha^{*} ; \pi_{e}\right)\left(\alpha^{*}-\alpha_{*}\right)>0 .
$$

The function $\varphi\left(\cdot ; \pi_{e}\right)$ has an inflection point at $\alpha=\alpha^{*}$ and a minimum at $\alpha=\alpha_{*}$. If $\mathscr{B}^{*}$ and $\mathscr{B}_{*}$ are balls of radii $\alpha^{*}$ and $\alpha_{*}$, respectively, then $\mathscr{B}^{*}$ is unstable, while $\mathscr{B}_{*}$ is stable.

Figure 1 illustrates the graphs of the first derivative $\varphi_{\alpha}^{\prime}$ for two functions $\varphi$ having such properties. The function plotted in Fig. 1a has $\alpha_{*}<\alpha^{*}$, while that of Fig. 1b has $\alpha_{*}>\alpha^{*}$. By using (1), we have

$$
D_{\pi_{e}}\left(\varphi_{\alpha}^{\prime}\left(\alpha ; \pi_{e}\right)\right)=4 \pi \alpha^{2}>0, \quad \text { for every positive } \alpha \text { and any } \pi_{e} ;
$$

then, when $\pi_{e}$ is perturbed, the graphs of Fig. 1 change in the manner shown in Fig. 2., where a dashed line refers to the smallest pressure.

Let $\alpha_{*}<\alpha^{*}$ (see Figs. 1a, 2a). As the outside pressure increases, $\mathscr{B}_{*}$ changes into a stable ball $\mathscr{B}^{+}$of radius $\alpha^{+}<\alpha_{*}$, while $\mathscr{B}^{*}$ is destroyed. This means that a positive perturbation of $\pi_{e}$ induces a dynamical instability of $\mathscr{B}^{*}$ which collapses toward $\mathscr{B}^{+}$. On the other hand, when the outside pressure is decreased, the functional $\mathscr{E}_{*}$ has three stationary balls, $\mathscr{B}_{1}, \mathscr{B}_{2}$, and $\mathscr{B}_{3}$, whose radii are, respectively, $\alpha_{1}, \alpha_{2}$, and $\alpha_{3} . \mathscr{B}_{1}$ and $\mathscr{B}_{3}$ are stable and, in particular, $\mathscr{B}_{1}$ is the absolute minimizer of $\mathscr{E}_{*}$; on the contrary, $\mathscr{B}_{2}$ is unstable. Thus, a decrement of the outside pressure produces a bifurcation of the unstable ball $\mathscr{B}^{*}$ into a stable and an unstable ball, close to each other as long as the perturbation of the pressure is small.

A similar analysis applies when $\alpha_{*}>\alpha^{*}$ (see Figs. 1b, 2b). In contrast to the previous instance, here the dynamical instability of $\mathscr{B}^{*}$ takes place when $\pi_{e}$ is decreased, and $\mathscr{B}^{*}$ bifurcates when $\pi_{e}$ is increased. 

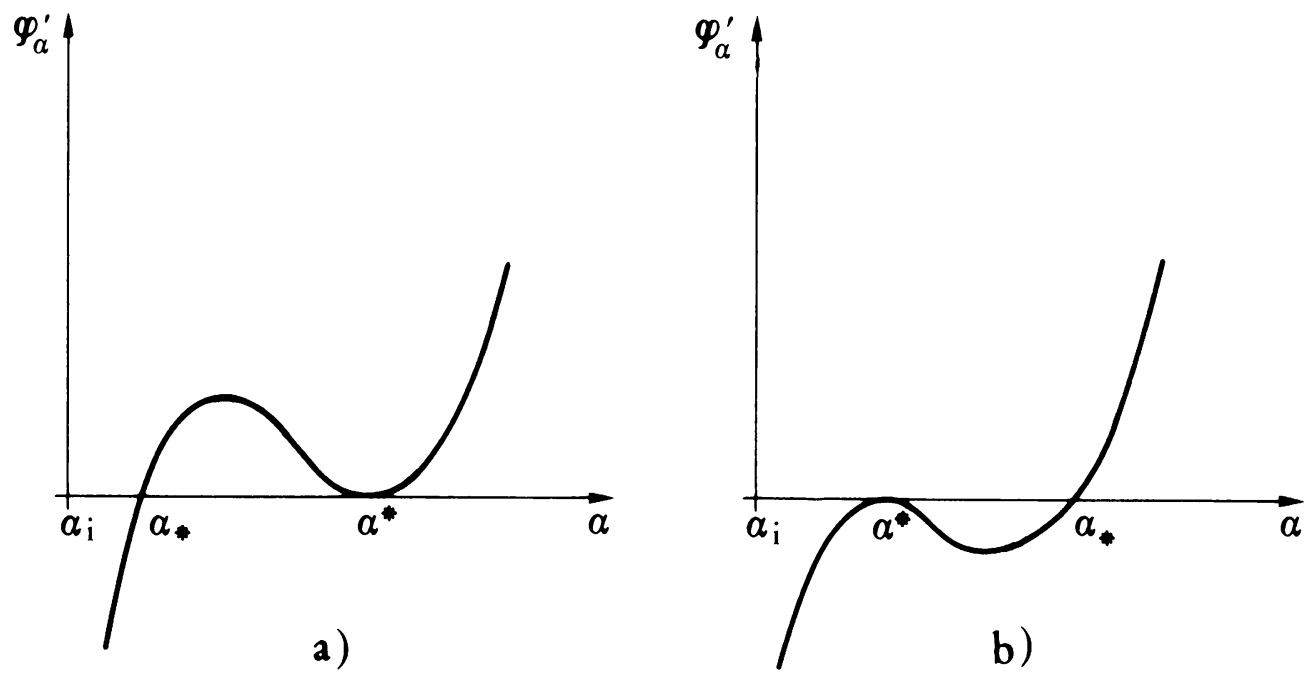

FIG. 1.
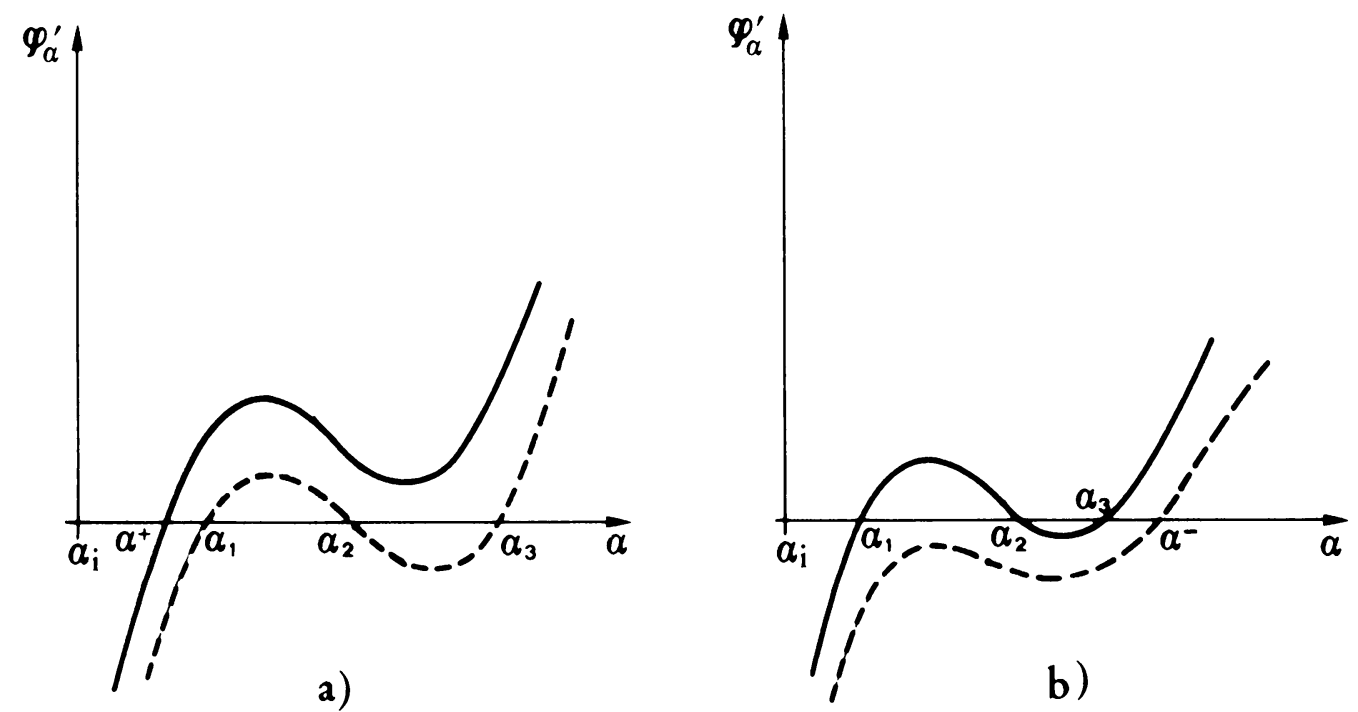

FIG. 2.

I shall show in the next section that the phenomenon described here actually occurs, as Eqs. (3) and (4) may have a common root. However, there are instances in which (3) and (4) are not compatible. For example, suppose that the function $\sigma$ is uniformly convex, i.e.,

$$
\sigma^{\prime \prime}(\nu) \geq \sigma_{0}>0, \quad \text { for every } \nu>0 .
$$

It easily follows from (1) that if $\mu$ is such that

$$
\mu<2 \sigma_{0}\left(3 \beta_{i}\right)^{4 / 3} / \tau_{e}(4 \pi)^{1 / 3},
$$

for a given $\beta_{i}$, then $\varphi_{\alpha}^{\prime \prime}\left(\alpha ; \pi_{e}\right)>0$ whenever $\varphi_{\alpha}^{\prime}\left(\alpha ; \pi_{e}\right)=0$. Thus, if the mass of $\mathscr{F}$ is sufficiently small, every stationary ball is stable. 
5. Inverse bubbles. If $\mathscr{F}_{i}$ and $\mathscr{F}_{e}$ are the same incompressible fluid and $\mathscr{F}$ is a gas, then $\mathscr{F}_{i}, \mathscr{F}_{e}$, and $\mathscr{F}$ form an inverse bubble (cf., e.g., [DR], p. 416). Experiments have shown that inverse bubbles may be very stable [B]. We shall see in this section that, under suitable assumptions on the constitutive properties of $\mathscr{F}$, the simplified model worked out here also predicts metastable equilibria for an inverse bubble.

Let $\tau$ be the common value of $\tau_{i}$ and $\tau_{e}$. We assume that the potential for $\mathscr{F}$ has the special form

$$
\sigma(\nu)=\chi \nu^{-(\xi-1)},
$$

where $\chi$ and $\xi$ are constants such that

$$
\chi(\xi-1)>0 \text { and } \xi>0 .
$$

If

$$
\xi<1,
$$

then Eq. (1) represents a polytropic real gas; otherwise (1) represents a thermally isolated perfect gas. Note that, by (2), Eq. (1) satisfies hypothesis $(\mathrm{H})$.

By using (4.1), it is easily seen that equations (4.3) and (4.4) are equivalent to

$$
\omega(\nu)=0, \quad \alpha=\left(\frac{\mu \tau}{2 \pi \sigma^{\prime \prime}(\nu)}\right)^{1 / 4}, \quad \beta_{i}=4 \pi \alpha^{3} / 3-\mu \nu,
$$

where the function $\omega: \mathbf{R}^{+} \rightarrow \mathbf{R}$ is defined as

$$
\omega(\nu):=\sigma^{\prime}(\nu)+2 \tau\left(\frac{2 \pi \sigma^{\prime \prime}(\nu)}{\mu \tau}\right)^{1 / 4}+\pi_{e} .
$$

For every root $\tilde{\nu}$ of $(4)_{1},(4)_{2}$ gives the radius of a metastable ball $\mathscr{B}^{*}$ and $(4)_{3}$ a compatible value of the volume of $\mathscr{B}_{i}$. By combining Eqs. $(4)_{2}$ and $(4)_{3}$, we get

$$
\beta_{i}=\eta(\nu) \text {, }
$$

where $\eta: \mathbf{R}^{+} \rightarrow \mathbf{R}$ is the map

$$
\eta(\nu):=(4 \pi / 3)\left(\frac{\mu \tau}{2 \pi \sigma^{\prime \prime}(\nu)}\right)^{3 / 4}-\mu \nu .
$$

As $\beta_{i}>0$, it follows from (6) that solutions of (4) may exist only if there are roots $\tilde{\nu}$ of $(4)_{1}$ such that

$$
\eta(\tilde{\nu})>0 .
$$

By inserting (1) into (5), it is a simple matter to verify that (4) 1 has only a root $\tilde{\nu}$ and that the function $\omega$ has the graph plotted in Fig. 3. On the other hand, it follows from (7) that, when $\sigma$ is given by (1), $\eta(\nu)$ is positive whenever

$$
\nu>\nu_{0}:=\left[\left(\frac{3 \mu}{4 \pi}\right)^{4}\left(\frac{2 \pi \chi \xi(\xi-1)}{\mu \tau}\right)^{3}\right]^{1 /(3 \xi-1)},
$$

so that (8) is satisfied if and only if $\omega\left(\nu_{0}\right)<0$ (see Fig. 3). An easy computation shows that

$$
\omega\left(\nu_{0}\right)=\pi_{e}-v \mu^{\delta},
$$

where

$$
\delta=-\xi /(3 \xi-1),
$$




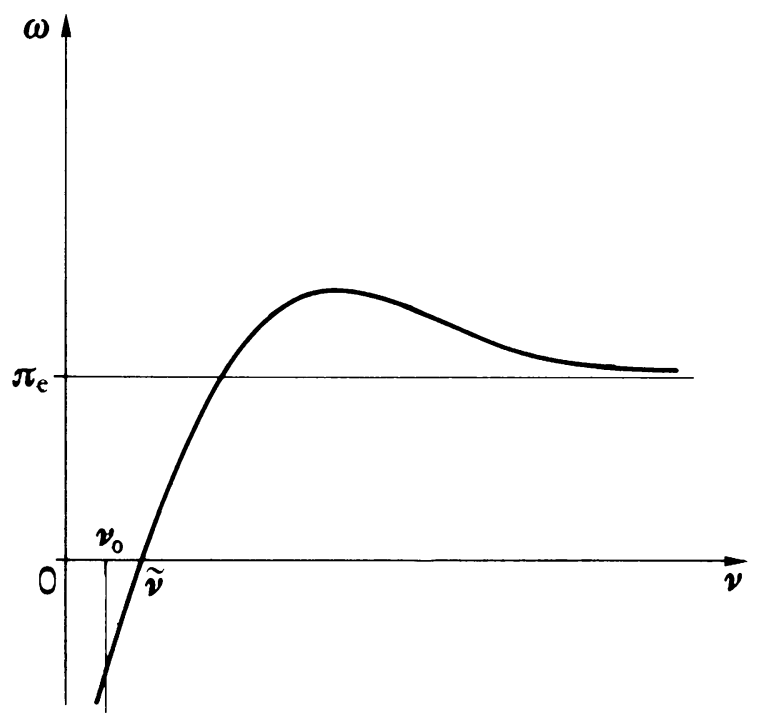

Fig. 3.

and $v$ is a constant depending on $\tau, \chi$, and $\xi$. Moreover, if

$$
\xi<1 / 3
$$

then $v$ and $\delta$ are positive, so that, by (10), there is a positive number $\mu_{c}$, which depends on $\tau, \chi, \xi$, and $\pi_{e}$, such that $\omega\left(\nu_{0}\right)<0$ for every $\mu>\mu_{c}$. This proves that Eqs. (4) are compatible and that the inverse bubble described here exhibits a metastable equilibrium configuration, provided the quantity of gas trapped in the fluid is sufficiently large.

By comparing (12) with (3) it turns out that an inverse bubble cannot be metastable if the inside gas is a thermally isolated perfect gas. Likewise, it is possible to show that there is no solution of (4) if (1) is replaced by

$$
\sigma(\nu)=-\chi \log \nu, \quad \chi>0,
$$

which represents a perfect gas at constant temperature.

Acknowledgments. I wish to thank Professors Jerry L. Ericksen and Morton E. Gurtin for their valuable help in writing this paper. This work was done while the author was visiting the Institute for Mathematics and Its Applications, University of Minnesota, and the Carnegie-Mellon University. The kind hospitality of these universities and the support of the Italian National Research Council are acknowledged.

\section{REFERENCES}

[A1] A. D. Alexandrov, Uniqueness theorems for surfaces in the large, V, Vestnik Leningrad Univ. 13, 5-8 (1958), English translation, Amer. Math. Soc. Transl. 21, 412-413 (1962)

[A2] A. D. Alexandrov, A characteristic property of spheres, Ann. Mat. Pura Appl. 58, 303-315 (1962)

[B] M. H. I. Baird, The stability of inverse bubbles, Trans. Faraday Soc., 56, 213-219 (1960)

[DG1] E. De Giorgi, Su una teoria generale della misura $(r-1)$-dimensionale in uno spazio ad $r$ dimensioni, Ann. Mat. Pura Appl. (4) 36, 191-213 (1954) 
[DG2] E. De Giorgi, Nuovi teoremi relativi alle misure $(r-1)$-dimensionali in uno spazio ad $r$ dimensioni, Ricerche Mat. 4, 95-113 (1955)

[DG3] E. De Giorgi, Sulla proprietà isoperimetrica dell'ipersfera, nella classe degli insiemi aventi frontiera orientata di misura finita, Mem. Accad. Naz. Lincei, Cl. Sci. Fis. Mat. Natur. 5, 33-44 (1958)

[DR] J. T. Davies and E. K. Rideal, Interfacial phenomena, Academic Press, New York, 1961

[FF] H. Federer and W. Fleming, Normal and integral currents, Ann. Math. 72, 458-520 (1960)

[FR] W. Fleming and R. Rishel, An integral formula for total gradient variation, Arch. Math. 11, 218$222(1960)$

[Gi] E. Giusti, Minimal surfaces and functions of bounded variation, Birkhauser, Boston, 1984

[GM] M. E. Gurtin and A. I. Murdoch, A continuum theory of elastic material surfaces, Arch. Rat. Mech. Anal. 57, 291-323 (1975)

[Gu] M. E. Gurtin, An introduction to continuum mechanics, Academic Press, New York, 1981

[H] H. Hopf, Über Flächen mit einer Relation zwischen den Hauptkrümmungen, Math. Nachr. 4, 232-249 (1950-1951)

[HLP] G. H. Hardy, J. E. Littlewood, and G. Polya, Inequalities, Cambridge University Press, Cambridge, 1952

[La] H. Lamb, Hydrodynamics, Dover, New York, 1945

[Li] G. H. Liebmann, Ueber die Verbiegung der geschlossenen Flächen positiver Krümmung, Math. Ann. 53, 81-112 (1900)

[O] R. Ossermann, The isoperimetric inequality, Bull. Amer. Math. Soc. 84, 1182-1237 (1978)

[R1] R. C. Reilly, Applications of the integral of an invariant of the Hessian, Bull. Amer. Math. Soc. 82, 579-580 (1976)

[R2] R. C. Reilly, Applications of the Hessian operator in a Riemannian manifold, Indiana Univ. Math. J. 26, 459-472 (1977)

[T] G. Talenti, Best constant in Sobolev inequality, Ann. Mat. Pura Appl. 110, 353-372 (1976) 